\title{
$I-20560$
}

Dry ofiti-x

UCID--20299

DE85 010030

RTMS-II OPERATIONS GUIDEBOOK

Dale H. Heikkinen

Apri1 1; 1985

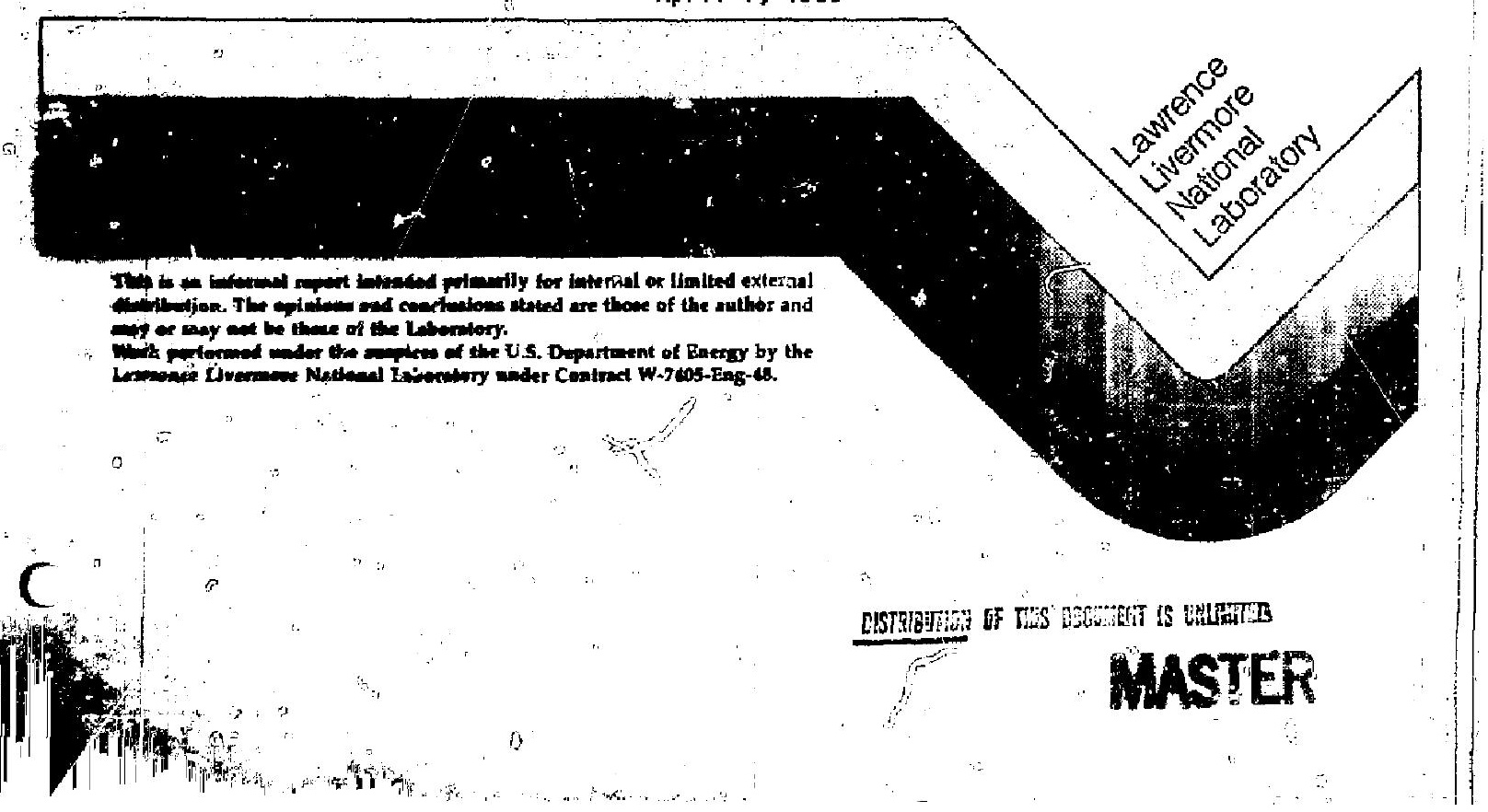




\section{RTNS-II OPERATIONS GUIDEBOOK}

by

Dale w. Heikkinen

\section{DISCLAIMER}

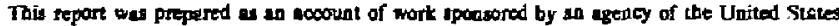

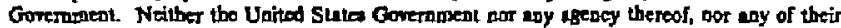

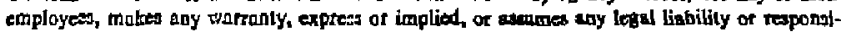
bility for the occuracy, completeness, or wefuloes of any isformation, apparatus, prudact, or proces disclosed, or represents that its une would not infringe privately owned righes. Reler

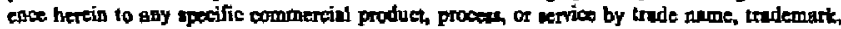

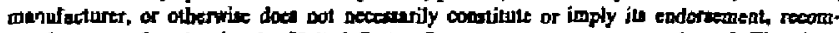
mendation, or fevoring by the Uniled Staten Government or eny agency therear. The views

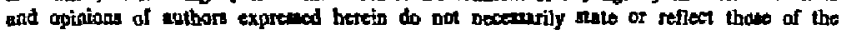
United states Government of any agency thereor. 
Section $A$. Introduction

Section B. Operator Training Program at RINS-II

Section C. Guide to Function, Location, and control of Water Valves, vacuum Valves, Thermocouple Gauges, Ion Gauges and Water Flow Switches

Section D. Guide to Interlocks and Interlock Chains

Section E. Guide to Remote Area Monitoring (RAM) Units

Section F. Guide to Neutron Source Alarms

Section G. Periodic Checks/Maintenance

Section $\mathrm{H}$. Loss of Vacuum

Section I. Use of Anti-Contamination Clothing

Section J. Tritiun scrubber Maintenance

Section K. Typical Neutron Source Start-up Procedure

Section L. Tritiun Monitoring System

Section M. Summary of Normal Settings/Readings

Section N. Additional Reading Material 
Section A

Introduction

February 22, 1985

4 


\section{INIRODUCTION}

A. This guidebook is intended primarily for use by the operations group at RTis-II. It is intended to provide training criteria, procedures and guidelines for operation of the RINS-II neutron sources and ancilliary equipment. Use of this document reguires full knowledge of the RUNS-II Facility Safety Procedure (FSP) and any Operational Safety Procedures (OSP) in effect. The RINS-II FSP defines the hazards which may be encountered at RINS-II and defines the procedures which must be followed is performing any task including operations.

The purpose of this document is to provide a central source of detailed information concerning systems and eguipment used in operating the RIts-II neutron sources on a day-to-day basis. All members of the Operations Group are expected to be familiar with its contents. It is also intended to be used in training new members of the operations Group.

Each section of this guidebook is dated individually and is subject to revision at any time. Section II supercedes UCID I466.

B. This guidebook was written with the help of the RINS,-II staff. I would like to acknowledge the help of the Electronics and Operations Groups in particular. 
Section B

Operator Training Program at

RINS-II

February 22, 1985

N: 
The training program for candidate accelerator operators at RINS-II has five parts.

I. Orientation

II. Instruction

III. Observation of Operations

IV. Supervised Operations

v. Demonstration of Skills and Vertification for Operations

I. ORIENJ'ATION

Orientation will first consist of a building tour and description of the facility. During orientation the candidate will become familiar with the material in the RINS-II FSP, and all other procedures and guicielines relevant to RINS-II safety and operations contained in the FINS-II operations Guidebook. Briefings or lectures may be used in place of or supp?ement the above. The following areas of information will be covered;

A. Interlock systems

1. Persannel safety interlock system

2. Water interlock system

3. Target interlock system

4. Vacuum interlock system

5. High voltage interlock systen

B. Radiation Monitoring systems

1. Tritium monitoring system

2. RAM system

3. Tritium swipe counting system

C. Rotating Target Assembly

1. 50-cm targets

2. Vacuum seal and associated equipment

3. Target controller

4. Chilled water system

D. Personnel Dosimetry

1. THD's

2. Bioassay

3. Radiation detection instruments

a) Induced activity detection

b) Tritium detection

c) Alarming dosimeters

4. Doselog and release form

5. Whole body counting 
E. Anti-Contanination Protection

1. Protective clothing

2. Respirators

3. Authorization and sign-in reguirements

F. Radicactive Materials

1. Handling

2. Storage

3. Movement within the RINS-II PaAA

4. Disposal

G. Tritium Scrubber System

1. Operation

2. Maintanance

3. Molecular Sieve Disposal

H. Personnel Access Control

1. RMA

2. Interlocked areas

II. INSTRUCTION

A. Courses Recpired (or have demonstrated equivalent experience)

1. H.S. 660 - Radiation Detection Instruments

2. H.S. 569 - Crane Safety

3. H.S. 160 - Emergency Aid

4. H.S. 601 - Radiation Safety orientation

5. H.S. I64 - Carōiopulmonary Resuscitation

B. Courses Required (before working on open target systeni)

I. H.S. 442 - Half- and Full-Face Mask Respirator Training

C. Courses - Optional (but recommended)

1. H.S. 462 - Respirator Protection

2. H.S. 562 - Fork Truck Safety

3. H.S. 520 - Laser Safety

4. H.S. 168 - Fire Extinguisher

5. H.S. 522 - Electrical Hazards Awareness

6. H.S. 503 - Pressure Safety Orientation

D. Each candidate will attend the Radiation Safety Lecture for the RINS-II facility. (An individual briefing may replace this requirement.:

E. Each candidate will complete a course in vacuun technology or have equivalent experience.

F. Each candidate will beccme tnowledgable in vacuum leak detection methods or have demongtrated experience.

G. Each candidate will be briefed on neutron source theory, and on the rotating target assembly. 


\section{OBSERVATION OF OPERATIONS}

Each candidate will observe operation of the neutron sources and auxiliary equipment for a minimum period of forty hours. During this period, the candidate will become familiar with the following:

A. Ion Source Operation

1. Start-up

2. Shut-down

3. Control and tuning parameters

4. Interlocks/alarms

5. Periodic maintenance/checks

6. Vacuum system

B. Haefely Equipment

1. Start-up

2. Shut-down

3. Periodic maintenance/checks

4. Interlocks/alarms

C. Beam 'rransport System
1. Interlocks

2. Control and tuning parameters

3. Vacuun system

4. Periodic maintenance/checks

D. Rotating Target system

1. Start-up

2. Shut-down

3. Interlocks/alarms

4. Vacuun system (including differential pumps)

5. Target controllex (rotation and scan)

6. Target-chilled water systern

7. Oil mister

8. Target change procedures

9. Períodic maintenarce/checks

E. Tritium Scrubber System

1. Operation

2. Periodic maintenance/checks

3. Alarms

F. Data Recording System

1. Start-up (ND and CAMAC)

2. Shut-down (ND and CANAC)

3. Paraneter entry and change

4. Neutron monitoring 
G. LOW Cooling System

1. Interlocks/alatms

2. Periodic maintenance/checks

H. Interlock System Check

I. Radioactive Materials

1. Proper handling and movement

2. Proper storage

3. Proper disposal

4. Decontamination

5. Anti-contamination protection

6. Swipe ccunting

7. Radiation surveying

8. Waste water system

J. Tritiun fonitoring

1. Exhalust fans

2. Monitors

3. Alarms

4. Periodic maintenance/checks

R. Experiment Set-up

TV. SUPERVISED OPERATING

Each candidate will operate the neutron source and auxiliary system for a minimum of two weeks under close supervision of a qual.ified operator. This period is to be "hands on".

v. DEMONSTRATION OF SKILIS ANE CERTIEICATION

The Accelerator Physicist and Lead Operator will observe the candidate during the above-mentioned "hands on" training and make a determination of qualification or necessary extension of the training period. Certification will be docunented and a copy placed in the Bldg. 292 files. Certification is subject to review at any time. Any operator who is inactive at RIMS-II for a period of one year must be receitified. 


\section{Section C}

RINS-II Guide to Function, Location and Control of:

Water Valves

Vacuum valves

Thermocuuple Gauges

Ion Gauges

Water Flow Switches

Febriary 22, 1985 


\begin{tabular}{|c|c|c|c|}
\hline & & & \\
\hline Label & Function & control & Phusical \\
\hline v1 & Ion source watex valve & Term water map & $\begin{array}{l}\text { Bend mag stand } \\
\text { TerTil. }\end{array}$ \\
\hline v2 & Bend mag water valve & Term liat $r$ map & $\begin{array}{l}\text { Bend mag stand } \\
\text { Terra. }\end{array}$ \\
\hline v3 & $\begin{array}{l}\text { Source and plenum tirbo } \\
\text { pump water valve }\end{array}$ & $\begin{array}{l}\text { Term turbo perre } \\
\text { controls }\end{array}$ & $\begin{array}{l}\text { Bend mag stana } \\
\text { Term. }\end{array}$ \\
\hline V11 & LCW supply $:$ o terminal & $\begin{array}{l}\text { Control Room } \\
\text { water map }\end{array}$ & Below Mezz. \\
\hline v12 & Lot recurn from terminal & $\begin{array}{l}\text { Control room } \\
\text { water map }\end{array}$ & Be? ow Mezz. \\
\hline v3 & LOW supply to beam tran. sys. & $\begin{array}{l}\text { Con'crol Roctin } \\
\text { water map }\end{array}$ & Below Mezz. \\
\hline V14 & LCW return from beam trans. sys. & $\begin{array}{l}\text { Control Rocan } \\
\text { water map }\end{array}$ & Below Mezz. \\
\hline v15 & LCW supply to mezzanine turbos & $\begin{array}{l}\text { Turbo control } \\
\text { Control Room }\end{array}$ & On Mezz. \\
\hline v16 & LCi supply to Target Roor. turbos & $\begin{array}{l}\text { Turbo control } \\
\text { Control Ruom }\end{array}$ & Above Mezz. \\
\hline v77 & LON supply to target cart & $\begin{array}{l}\text { Control Roosn } \\
\text { water map }\end{array}$ & Above Mezz. \\
\hline V18 & LOW return from target cart & $\begin{array}{l}\text { Contral Room } \\
\text { water map }\end{array}$ & Above Mezz. \\
\hline v9 & Ncc used & & \\
\hline V2G & Wot used & & \\
\hline V21 & Not used & & \\
\hline $\mathrm{v} 22$ & Target chilled water retuln & $\begin{array}{l}\text { Control Rnom } \\
\text { target chain }\end{array}$ & Below Rezz. \\
\hline v23 & Target chilled water supply & $\begin{array}{l}\text { Control Room } \\
\text { target chain }\end{array}$ & Above Mezz. \\
\hline
\end{tabular}


Vacuum Valves

\begin{tabular}{|c|c|c|c|}
\hline \multirow[b]{2}{*}{ Iabel } & \multirow[b]{2}{*}{ Runction } & \multicolumn{2}{|c|}{ Iocation } \\
\hline & & Control & Phusical \\
\hline $\mathbf{v}$ & Roughing by-pass - source turbo & Terminal vac map & Term. \\
\hline vla & Roughing by-pass - Plenum turbo & Terminal vac map & Eoreline Term. \\
\hline V2 & Source turbo fore vac valve & Terminal vac map & Foreline Term. \\
\hline$\sqrt{3}$ & $\begin{array}{l}\text { Source and plenum turbo } \\
\text { auto vent valve }\end{array}$ & $\begin{array}{l}\text { Turbo puris } \\
\text { controllers }\end{array}$ & On solurce turbo \\
\hline $\mathrm{v4}$ & Beam line gate valve & Terminal vac map & $\begin{array}{l}\text { Below ion source } \\
\text { Term. }\end{array}$ \\
\hline v5 & Bean line gate valve & $\begin{array}{l}\text { Terminal and } \\
\text { Control Room } \\
\text { vac maps }\end{array}$ & $\begin{array}{l}\text { Accel. tube entrance } \\
\text { Term. }\end{array}$ \\
\hline v6 & Vent valve source & Terminal vac map & $\begin{array}{l}\text { Bending mag chamber } \\
\text { Term. }\end{array}$ \\
\hline$\eta$ & Vent valve - Term. Corepump & Terminal vac map & Foreline rerrn. \\
\hline v8 & Source gas valve & $\begin{array}{l}\text { Terminal and ion } \\
\text { source control } \\
\text { panel }\end{array}$ & Hot rack Term, \\
\hline V10 & Source vent valve & Manual & Ion source Term. \\
\hline V11 & Scurce roughing valve & Manual. & Foreline Term. \\
\hline $\mathrm{V} 12$ & Plenum turbo fore vac valve & Terminal vac map & Foreline Term, \\
\hline V15 & Accel, tube roughing by-pass & $\begin{array}{l}\text { Control Room } \\
\text { vac map }\end{array}$ & On Mezz. \\
\hline V16 & TP1 turbo fore vac valve & $\begin{array}{l}\text { Control froon } \\
\text { vac map }\end{array}$ & On Mezz. \\
\hline $\mathrm{V} 17$ & TP2 turbo fore vac valve & $\begin{array}{l}\text { Control Room } \\
\text { vac map }\end{array}$ & On Mezz. \\
\hline $\mathrm{v} 1$ & Beam line gate valve & $\begin{array}{l}\text { Control Room } \\
\text { vac map }\end{array}$ & On Mezz. \\
\hline V19 & Target Roon turbo fore vac valve & $\begin{array}{l}\text { Control Room } \\
\text { vac map }\end{array}$ & Below Mezü. \\
\hline V20 & TP3 turbo fore vac valve & $\begin{array}{l}\text { Cantrol roon } \\
\text { vac map }\end{array}$ & Target Room \\
\hline v21 & IP4 turbo fore vac valve & $\begin{array}{l}\text { Control Room } \\
\text { vac map }\end{array}$ & Target Room \\
\hline v22 & Target roughing valve & $\begin{array}{l}\text { Control Room } \\
\text { vac map }\end{array}$ & Below ME'zz. \\
\hline
\end{tabular}


$C+3$

vacuum valves

\begin{tabular}{|c|c|c|c|}
\hline \multirow[b]{2}{*}{ Iabel } & \multirow[b]{2}{*}{ Function } & \multicolumn{2}{|c|}{ Iocation } \\
\hline & & Control & Ehysical \\
\hline$\sqrt{23}$ & Beam line gate vaive & $\begin{array}{l}\text { Control Rocm } \\
\text { vac map }\end{array}$ & Target Room \\
\hline V24 & Vacuum pump exhaust to stack & $\begin{array}{l}\text { Control Rocm } \\
\text { vac map }\end{array}$ & Below Mezz. \\
\hline v25 & Vaculen pump exhaust to sarubicer & $\begin{array}{l}\text { Cantrol Room } \\
\text { vac map }\end{array}$ & Below Mezz. \\
\hline v26 & TP1, TP2, auto vent valve & $\begin{array}{l}\text { Turbo pump } \\
\text { controllers }\end{array}$ & On TP1 \\
\hline v27 & TP3, TP4 auto vent valve & $\begin{array}{l}\text { Turbo pump } \\
\text { controllers }\end{array}$ & On TP3 \\
\hline v28 & Vent valve - accel. tube & $\begin{array}{l}\text { Control Room } \\
\text { vac map }\end{array}$ & On Mezz. \\
\hline v29 & Vent valve - beam transport & $\begin{array}{l}\text { Control Room } \\
\text { vac map }\end{array}$ & On Mezz. \\
\hline v30 & Vent valve - Earget & $\begin{array}{l}\text { Control Room } \\
\text { vac map }\end{array}$ & Below Mezz. \\
\hline V31 & Vent valve - fore puit & $\begin{array}{l}\text { Control froom } \\
\text { vac map }\end{array}$ & Below Mezz. \\
\hline v32 & Roughing bypass - target & $\begin{array}{l}\text { Control Roon } \\
\text { vac map }\end{array}$ & Below Mezz. \\
\hline v33 & Roughing bypass - beam transport & $\begin{array}{l}\text { Control Roon } \\
\text { var siap }\end{array}$ & Below Mezz. \\
\hline
\end{tabular}

Term. = High Voltage Terminal - Machine Room

Mezz. = Mezzanine - Machine Room 


$$
\text { c-4 }
$$

Thermocouple Gauges

\begin{tabular}{ll} 
Iabel & \multicolumn{1}{c}{ function } \\
TC1 & Fore line TC gauge \\
TC2 & Source turbo TC gauge \\
TC3 & Ion source TC gauge \\
TC4 & Plenum turbo TC gauge \\
TC6 & Beam trans. sys. forepump \\
TC7 & TC gauge \\
TC8 & Beam trans, sys. TC gauge \\
TC9 & TP3, TP4 foreline TC gauge \\
TC10 & Target TC gauge \\
TC11 & Target roughing line TC gauge \\
TC26 & Outer differentia 1 groove \\
TC27 & Inner differenti tl groove \\
\hline TErm. = High Voltage Terminal - Machine Room &
\end{tabular}

\begin{tabular}{ll}
\hline Control & Focation. \\
\hline Terminal & Foreline Term. \\
Terminal & Ion source Term. \\
Terminal & Foreline Term. \\
Terininal & Below Mezz. \\
Control Room & \\
Control Room & On Mezz. \\
Control Roon & On Mezz. \\
Control Room & Below Mezz. \\
Control Room & Target Room \\
Control Room & Below Mezz. \\
Control Room & On Mezz. \\
Control Room & On Mezz.
\end{tabular}


$c-5$

Ion Gauges

Iabel

Function

IGI

IG2

IG3

IG4

IG5

Target Roon IG

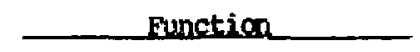

Ion source turbo IG

Term. plenum turbo IG

Accelerator tube IG

Beam transport system IG
Control Iocation Phusical

Terminal

Terminal

Terminal

Terminal

Control Room

On Hezz.

Control Roan

On Hezz.

Control Roam

Target Rocrn

Mezz. = Mezzanine - Machine Room 


\begin{tabular}{|c|c|c|c|}
\hline \multirow[b]{2}{*}{ Label } & \multirow[b]{2}{*}{ Eunction } & \multicolumn{2}{|c|}{ location } \\
\hline & & Control & Phurgical \\
\hline FS2 & Cathode & $\begin{array}{l}\text { Water map } \\
\text { Term. }\end{array}$ & $\begin{array}{l}\text { Ion Source Stand } \\
\text { Term. }\end{array}$ \\
\hline FS3 & Zwischen electrode & $n$ & $\pi$ \\
\hline FS4 & Ancole electrode & $n$ & $n$ \\
\hline PS5 & Source \& shaping mag/reflector & n & $n$ \\
\hline ES6 & Accel/Decel/Gind electrode & $\pi$ & $n$ \\
\hline FS7 & Bend tnag. coils \& chamber & $\pi$ & $n$ \\
\hline FS8 & Bend mag liner, $0^{\circ}$ and $45^{\circ}$ targets & " & $n$ \\
\hline FS9 & Solenoid lens & $n$ & $"$ \\
\hline FS10 & $\begin{array}{l}\text { Accel. tube entrance elect. and } \\
\text { plunging target }\end{array}$ & $n$ & $n$ \\
\hline Fsll & source \& plenum turbos & " & $n$ \\
\hline FS12 & TP1, TP2 turbo pumps & $\begin{array}{l}\text { Control Room } \\
\text { water map }\end{array}$ & On Mezz. \\
\hline $\operatorname{FSI3}$ & Anode accel. col. & n & n \\
\hline FSI4 & Plenum collimator & " & $n$ \\
\hline FS215 & Mezzanine guad beam pipe & $"$ & n \\
\hline FS16 & Camera box & $n$ & $n$ \\
\hline FS17 & Wall beam pipe TG Room guad & $"$ & $"$ \\
\hline ESI 8 & Copper pipe & $n$ & $\pi$ \\
\hline FS19 & Accelerator tube & $"$ & $"$ \\
\hline FS20 & TP23, TP4 turbo pandp & $n$ & Below Mezz. \\
\hline FS21 & Target cart water & $n$ & On Mezz. \\
\hline TOW Flont & Target chilled water & On Hezz. & Control Roor \\
\hline
\end{tabular}


Section D

Guide to Interlocks and Interlock Chains

February 22, 1985

क्ष: 


\section{INIRODUCTION}

The guides on the following pages describe the interlocks and interlock chains in use on the RJNS-II neutron sources. The description gives the interlocking element (what does the interlocking), the interlocked element (what is interiocked), the effect of the interlock (i.e. permissive, close valve, etc.), and the type of interlock (MP = primarily for neutron source protection, EP = primarily for perscrunel protection). Only the primary interlocked elements are given (i.e. an interlocking element may have an effect on several elements).

In any display panel containing interlock indicators, the following convention nolds

white light red light amber light green Iight
- control power on

- interlocking element

- indicator light only

- interlock chain complete 
$D-2$

Guide to Water Interlocks (TerminaI)*

\begin{tabular}{|c|c|c|c|}
\hline $\begin{array}{l}\text { Interlocking } \\
\text { Qlement }\end{array}$ & $\begin{array}{l}\text { Interlocked } \\
\text { Element }\end{array}$ & $\begin{array}{l}\text { Effect of } \\
\text { Interlock }\end{array}$ & Tupe \\
\hline FSI & $\begin{array}{l}\text { Extraction power } \\
\text { supply }\end{array}$ & $\begin{array}{l}\text { Power supply } \\
\text { off }\end{array}$ & MP \\
\hline FS2 & $\begin{array}{l}\text { Hot rack power } \\
\text { Decel }\end{array}$ & $\begin{array}{l}\text { Power off } \\
\text { Power off }\end{array}$ & $\mathrm{MP} / \mathrm{PP}$ \\
\hline FSJ & $\begin{array}{l}\text { Hot rack power } \\
\text { Decel }\end{array}$ & $\begin{array}{l}\text { Power off } \\
\text { Power of } f\end{array}$ & MP/PP \\
\hline FS4 & $\begin{array}{l}\text { Hot: rack power } \\
\text { Decel }\end{array}$ & $\begin{array}{l}\text { Power off } \\
\text { Power off }\end{array}$ & MP/PP \\
\hline FS5 & $\begin{array}{l}\text { Hot racik power } \\
\text { Decel }\end{array}$ & $\begin{array}{l}\text { Power off } \\
\text { Power off }\end{array}$ & $\mathrm{MP} / \mathrm{PP}$ \\
\hline FS6 & $\begin{array}{l}\text { Hot rack power } \\
\text { Decel }\end{array}$ & $\begin{array}{l}\text { Fower off } \\
\text { Fower off }\end{array}$ & $M P / P P$ \\
\hline F57 & $\begin{array}{l}\text { Hot rasik power } \\
\text { Dercel }\end{array}$ & $\begin{array}{l}\text { Power off } \\
\text { Power off }\end{array}$ & $\mathrm{MP} / \mathrm{PP}$ \\
\hline FSB & $\begin{array}{l}\text { Hot rack power } \\
\text { Deceli }\end{array}$ & $\begin{array}{l}\text { Power off } \\
\text { Power off }\end{array}$ & $\mathrm{MP} / \mathrm{PP}$ \\
\hline FS9 & Leis magnet power & Power off & $\mathbf{M P}$ \\
\hline FS10 & $\begin{array}{l}\text { Hoí rack power } \\
\text { Decel }\end{array}$ & $\begin{array}{l}\text { Power off } \\
\text { Power off }\end{array}$ & $\mathrm{MP} / \mathrm{PP}$ \\
\hline ESll & $\begin{array}{l}\text { Source turbo punts } \\
\text { Plenum turbo punफs }\end{array}$ & $\begin{array}{l}\text { Turbo pumps off and } \\
\text { starts auto vent }\end{array}$ & $\mathbf{M P}$ \\
\hline
\end{tabular}


D-3

Guide to Vacuum Interlocks (Teminal)*

Interlocking

Element

TCl

TC2

TC3

$\mathrm{TC}_{4}$

IGI

IG2

V4*

V5*
Interlocked

glement.

V2, V12

V1, VIA

V2, V4

source plenum turbo

$\mathrm{H}_{2} \mathrm{O} \vee \mathrm{r}$

V4, v8

V12, V4

Source plenum turbo

$\mathrm{H}_{2} \mathrm{O} \quad \mathrm{V}$

v5

V5

Hot rack power

Bend magnet
Effect of

Interlock

Close V2, V12

Permissive to open

Close V2, V4

Turbos off \& start auto vent

close V3

Close V4, v8

Close V12, V4

Turbos off \& start

auto vent

close v3

Close v5

Close v5

Power off

Power off
$M P$

MP

MP

Type .

MP

MP

MP

MP

MP

MP

MP

*Part of terminal interlock chain 
Guide to Terminal Isolation Fack Interlock Chain*

\section{Interlacking \\ Flement.}

Ion source $\mathrm{H}_{2} \mathrm{O}$ Elow Summation of FS2-8, FS10

Bend magnet $\mathrm{H}_{2} \mathrm{O}$ flow

V4

Roof fans

Rermina: door closed

Door bypassed

(maintenance mode)
Interlocked
Element
Type

Isolation rack power Power off

MP

Isolation rack power Power off

MP

Isolation rack power Power off MP

rsolation rack power Power off MP

Isolation rack power Power off PP

None Indicator only PP

*Isolation. rack power on (cinain complete) has a 20-second delay to allow time to exit terminal and close door. 
$D-5$

Guide to Terminal Chain Indicator Lights*

Indicator Iabel

Decel power

Crontoar open

HV control on

Steerer power

Bend magnet power

Eend magnet $\mathrm{H}_{2} \mathrm{O}$ flow

V5 open

Beam line gate valves

Lens power

Lens $\mathrm{H}_{2} \mathrm{O}$ flow
Heaning of Indicator

Power on to Decel power supply

Crowbar to isolation rack open

Extraction/Decel switch at control console or

Power on to steerer power supplies

Power on to bend magnet power supply

Bend magnet $\mathrm{H}_{2} \mathrm{O}$ tilow switch made up

v5 gate valve open

v18, v23 gate valves open

Power to solenoid lens power supply

Solenoid lens Hzo flow switch made up

*hese lights are indicators only. Indicator lights are anber colored. 
Guide to Terminal High Voltage Interlick Chain

\begin{tabular}{|c|c|c|c|}
\hline $\begin{array}{l}\text { Interiocking } \\
\text { Element }\end{array}$ & $\begin{array}{l}\text { Interlocked } \\
\text { Elemert }\end{array}$ & $\begin{array}{l}\text { Effect of } \\
\text { Interlock }\end{array}$ & type \\
\hline $\begin{array}{l}\text { Accelerator } \\
\text { HzO flow } \\
\text { FS10, FSt }\end{array}$ & $\begin{array}{l}\text { Bend magnet } \\
\text { Isolation rack } \\
\text { Power }\end{array}$ & $\begin{array}{l}\text { Power off } \\
\text { Fower off }\end{array}$ & MP \\
\hline $\begin{array}{l}\text { Mode switch } \\
\text { Normal (key Aj }\end{array}$ & $\begin{array}{l}\text { Bend magnet } \\
\text { Solenoid lens } \\
\text { Hot rack crowbar } \\
\text { Decel } \\
\text { Cober power supply }\end{array}$ & $\begin{array}{l}\text { Power off } \\
\text { Pomer off } \\
\text { Crowbar } \\
\text { Pomer off } \\
\text { Output off }\end{array}$ & $\begin{array}{l}\text { MP } \\
\text { MP } \\
\text { PP } \\
\text { PP } \\
\text { DP }\end{array}$ \\
\hline Cober power supply & None & Indicator only & $P P$ \\
\hline Ground hook stowed & $\begin{array}{l}\text { Bend magnet } \\
\text { Solenoid lens } \\
\text { Hot rack crowbar } \\
\text { Decel power supply } \\
\text { Cober power supply }\end{array}$ & $\begin{array}{l}\text { Power off } \\
\text { Power off } \\
\text { Crowhars } \\
\text { Power off } \\
\text { Output off }\end{array}$ & $\begin{array}{l}\text { MP } \\
\text { PP } \\
\text { PP } \\
\text { PP }\end{array}$ \\
\hline Terminal door closed & $\begin{array}{l}\text { Bend magnet } \\
\text { Solenoid lens } \\
\text { Hot rack crowbar } \\
\text { Hot rack power } \\
\text { Decel power supply } \\
\text { Cober power supply_ }\end{array}$ & All power off & $\begin{array}{l}\text { MP } \\
\text { MP } \\
\text { PP } \\
\text { PP } \\
\text { PP } \\
\text { PP }\end{array}$ \\
\hline
\end{tabular}


Gide to Water Interlocks (Beam Transport)*

\begin{tabular}{|c|c|c|c|}
\hline $\begin{array}{l}\text { Interlocking } \\
\text { Eilement }\end{array}$ & $\begin{array}{l}\text { Interlocked } \\
\text { Element }\end{array}$ & $\begin{array}{l}\text { Effect of } \\
\text { Interlock }\end{array}$ & Type \\
\hline $\operatorname{ESL} 2 \star \pi$ & $\begin{array}{l}\text { TP1, TP2 } \\
\text { Baefely high voltage }\end{array}$ & $\begin{array}{l}\text { TP1, TP2 off } \\
\text { HV off }\end{array}$ & $\begin{array}{l}\text { MP } \\
\text { MP }\end{array}$ \\
\hline FS13** & Haefely high voltage & $\mathrm{HV}$ off & MP \\
\hline ES14** & Haefely high voltage & ET off & MP \\
\hline FS15** & Haefely high voltage & HV off & MP \\
\hline FS16** & Haefely high voltage & HV off & MP \\
\hline FSI $7 * *$ & Haefely high voltage & HV off & MP \\
\hline FSL18** & Haefely high voltage & HV offf & $\mathbf{M P}$ \\
\hline FSI9** & Haefely high voltage & HV off & MP \\
\hline FS20** & $\begin{array}{l}\text { TP3, TP4 } \\
\text { Haefely high voltage }\end{array}$ & $\begin{array}{l}\text { TP3, TP4 off } \\
\text { HV off }\end{array}$ & $\begin{array}{l}M P \\
M P\end{array}$ \\
\hline
\end{tabular}

* part of Operational interlock chain.

** Flow switch summation is part of pemissive to electron trap. 
Guide to Vaci:am Interlocks (Beam Transpert)

\begin{tabular}{|c|c|c|c|}
\hline $\begin{array}{l}\text { Interlocking } \\
\text { Elerent. }\end{array}$ & $\begin{array}{l}\text { Interlocked } \\
\text { EIement }\end{array}$ & $\begin{array}{l}\text { Effect of } \\
\text { Interlyok }\end{array}$ & TYT \\
\hline re7 & V16, V17, TP1, TP2, & $\begin{array}{l}(\mathrm{Close} \mathrm{V} 16, \mathrm{v} 7\rangle) \\
\text { TP1, TP2 off }\end{array}$ & MI \\
\hline $\begin{array}{l}\text { TP1, TP2 Controller } \\
\text { Start/Bypass }\end{array}$ & $\begin{array}{l}\mathrm{V} 16, \mathrm{~V} 17 \\
\mathrm{TPI}, \mathrm{TP2}\end{array}$ & $\begin{array}{l}\text { Pernissive to open } \\
\text { Permissive to start }\end{array}$ & $\frac{10}{10}$ \\
\hline $\begin{array}{l}\text { TC8 } \\
\text { TC9 } \\
\text { गP3, TP4 Controller } \\
\text { Start/Bypass }\end{array}$ & $\begin{array}{l}\text { TP3, TP4 } \\
\text { V19 } \\
\text { V19 } \\
\text { TP3, TP4 }\end{array}$ & $\begin{array}{l}\text { TP3, TP4 off } \\
\text { Close V19 } \\
\text { Pecmizsive to open } \\
\text { Permir-ive to start }\end{array}$ & $\begin{array}{l}\text { NF } \\
\mathrm{MP} \\
\mathrm{MP} \\
\mathrm{MP}\end{array}$ \\
\hline $\mathrm{TCll}$ & v22 & Permissive to open & MP \\
\hline IG3 & v $8 *$ & Close V18 & $\mathbb{M P}$ \\
\hline IG4 & $\mathrm{V} 18^{*}, \mathrm{~V} 23^{*}$ & Close v18, V23 & $\mathrm{MP}$ \\
\hline IG5 & $\mathrm{v} 23^{*}$ & Close v23 & $M D$ \\
\hline $\begin{array}{l}\text { Blower pressure } \\
\text { switcin }\end{array}$ & $\begin{array}{l}\text { TP1, TP2 } \\
\text { TP3, TP4 }\end{array}$ & $\begin{array}{l}\text { TP1, TP2 off } \\
\text { TP3 } 3, \text { TP4 off }\end{array}$ & MP \\
\hline $\begin{array}{l}3 \text { psi pressure } \\
\text { switch }\end{array}$ & V24 & $\begin{array}{l}\text { Opens } \sqrt{24} \text { to stack on } \\
\text { forepump. Exhaust } \\
\text { pressure high. }\end{array}$ & MP \\
\hline v22 & $\mathrm{v} 16, \mathrm{v} 7, \mathrm{v} 19^{-}$ & $\begin{array}{l}\text { Interlock element closes } \\
\text { upon openiny of inter- }\end{array}$ & MP \\
\hline V15 & $\mathrm{v} 15, \mathrm{~V} 7, \mathrm{v} 19$ ! & $\begin{array}{l}\text { locking element. Us: } \\
\text { caution on opening }\end{array}$ & MP \\
\hline $\begin{array}{l}\sqrt{32} \\
\sqrt{33}\end{array}$ & $\begin{array}{l}\text { v16, v17, v19 } \\
\text { v16, v17, v19_l }\end{array}$ & interlocking elenent & $\mathbb{M P}$ \\
\hline v18*, v23* & $\begin{array}{l}\text { daefely high voltage } \\
\text { bend magnet } \\
\text { Arc }\end{array}$ & $\begin{array}{l}\text { HV off } \\
\text { power off } \\
\text { Arc scram }\end{array}$ & $\begin{array}{l}\mathrm{MP} \\
\mathrm{MPP}\end{array}$ \\
\hline
\end{tabular}

*Part of operational interlock chain. 
D-9

Guide to Eigh Voltage Interlock Chain*

Interiocking

Elemant

Mainterance

ground hook stored.

a Grownd

hook stored

Machine Room

door closed

Target Room

door closed

in crontar closed

HV crowbar air

supply

HV crowbar open

*Permissive in operational interlock chain.

Haefely high voltage

Haefely high voltage
Effect of Interluck

Mype

HV off

PP/MP

Haefely high voltage HN off

PP/MP

Baefely high voltage HV off

Pp

EV off

PP/MP

Indicator light orly

PP/NP

Indicator light only

MP

HV off

PP/MP 
Guide to Run/Safe Interlock Chain

Interlocking

Element

Run/safe MR

HV pit north

Runtsafe MR

HV pit south

Run/safe MR

Mezzanine

Run/safe MR

southest

Ran/safe MR

south

Rin/safe MR

door

Run/safe TR

interior

Rin/safe TR

door

$\mathrm{Run} / \mathrm{safe}$ interlocks complete

\section{Interlocked}

Element:

Haefely high voltage

Arc

Haefely high voltage

Arc

Haefely high voltage

Arc

Haefely high voltage

Arc

Haefely high voltage

Arc

Haefely high voltage

Arc

Haefely high voltage

Arc

Haefely high voltage

Arc

Baefely high voltage

Arc

Operational interlock chain
Effect of

Interilock.

HV off

Arc scram

Type

PP

PP

HV off $\quad$ PF

Arc scram $\quad$ PP

HV off PP

Arc scram $\quad$ PP

HN off $\quad$ PP

Arc scram PP

HV off $\quad P P$

Arc scram PP

HV off PP

Arc scram PP

HV off PP

Arc scram* PP

HV off $\quad$ PP

Arc scram*

HV off

Arc scran* $\quad$ DP

permissive in $\quad$ PP

oper. interlock chain

\#Arc scrams if plunging target out or quadrupole power supplies off when Target Room door open. 
Guide to Operational Interlock Chain*

Interlocking
Element

$\mathrm{H}_{2} \mathrm{O}$ flow

beam transport

Gate valves

v18, v23

Run/safe**

Interlock chain

High voltagetћ*

interlock chain

Remote area monitor

power

Emergency switch****

Key A operate

Warning horn

(complete)

Rey B operate

operational interlocks complete
Interlocked

Element

Haefely high voltage Arc

Haefely High Voltage

Arc

bend mag

Haefely high voltage

Arc

Haefely high voltage

Are

Haefely high voltage

Arc

Haefely high voltage

Arc

Baefely high voltage Arc

Haefely high voltage Arc

Haefely high voltage

Haefely high voltage
Effect of

Interlock

Type

HV off

Arc scram

MP

MP

HV off

Arc scram

off

MP

MP

MP

HV off

Arc scram

PP

PP

HV off

Arc scran

HV off

Arc scram

HV off

Arc scram

PP

PP

HV off

Arc scram

PD

PP

HV off

Arc scram

PP

PP

HV off

Pp

HV off

PP

toperational interlock chain part of total interlock chain * Surmation of run/safe interlock chain

***Summation of high voltage interlock chain

****Switch breaks interlock chain but has no indicator light 
Guide to Target Interlock Chain*

\begin{tabular}{|c|c|c|c|}
\hline $\begin{array}{l}\text { Interlocking } \\
\text { Element }\end{array}$ & $\begin{array}{l}\text { Interlocked } \\
\text { Element }\end{array}$ & $\begin{array}{l}\text { Effect of } \\
\text { Interlock }\end{array}$ & Mree \\
\hline $\begin{array}{l}\text { Target cart } \\
\text { Water FS } 21\end{array}$ & $\begin{array}{l}\text { Target controller } \\
\text { (Rotation) }\end{array}$ & Rotation off & MP \\
\hline Oil mist & $\begin{array}{l}\text { Target controller } \\
\text { (Rotation) }\end{array}$ & Rotation off & MP \\
\hline Lift-off Āir & $\begin{array}{l}\text { Target controller } \\
\text { (Rotation) }\end{array}$ & Rotation off & MP \\
\hline $\begin{array}{l}\text { Target } \text { H }_{2} \mathrm{O} \\
\text { Flow (low) }\end{array}$ & $\begin{array}{l}\text { Target controllet } \\
\text { (Rotation) }\end{array}$ & $\begin{array}{l}\text { Rotation off } \\
\text { For low flow }\end{array}$ & $\mathbf{M P}$ \\
\hline $\begin{array}{l}\text { Target } \mathrm{E}_{2} \mathrm{O} \\
\text { Flow (high) }\end{array}$ & None & Alarm only & MP \\
\hline TCW PUIP & $\begin{array}{l}\text { Target controller } \\
\text { (Rotation) }\end{array}$ & Rotation off & $\mathbf{M P}$ \\
\hline TCW compressor \$I & None & Indicator light on ${ }^{\prime}$ & MP \\
\hline TCW cormpressor $\$ 2$ & None & Indicator light only & MP \\
\hline $\begin{array}{l}\text { Target rotation } \\
\text { High }\end{array}$ & Target controller & Rotation off & $\mathbf{M P}$ \\
\hline $\begin{array}{l}\text { Target rotation } \\
\text { Low }\end{array}$ & Haefely high voltage & HV off & MP \\
\hline
\end{tabular}

*Target interlock chain part of total interlock chain 
Guide to Total Interlock Chain

Interloking
Element

Opirational interlock chain conplete

High voltage interlock chain complete

Target interlock complete

Total interlocks complete
Interlocked

Blement

Haefely high voltage

Operational interlock chain

Haefely high voltage

Haefely high voltage
Effect of Interlock Type

ev off

PP

HV off

Ar: scram PP

HV off

MP

MP

HV off

PP 
Section E

Guide to Remote Area Monitoring (RAN) Units

February 22, 1985

8 
RAM unice for detecting prompt or induced radiation are located at the following locations:
A. Left Machine Room
B. Left Target Rocm door
C. Left Target Rocm door fence position
D. Power supply Room rack
E. Hot Work Room door
F. Target storage Vault door
G. Right Machine Room
H. Right larget Room door fence position

The local control units for the faMs are located in the control Room. These have logarithnic analogle meters which indicate radiation levels at the various locations. There are al on two or three alarm level adjustments which set the low, intermediate (if applicable) and high levels. Associated with these levels are amber, blue (if applicable), and red lights. The levels set are indicated by the appropriately colored dials. An amber light on a control Room unit means possible RAM unit failure and alarms in the Control Room (see Guide to Alams at RTnS-II). RAM units C, D, E, F and $H$ (see above) also have analogue readouts at the detector location (remote). They also have light indicators (amber and red) and an audible alarm. The audihle alarm is connected to tie red light. Units $C, D, E$ and $F$ indicate (at the remote location) intermediate levels by the amber light and high levels by the red light and audible alarm.

During normal operation (bearn to target) the Hachine Room RAM units will read high levels of radiation $(01000 \mathrm{mR} / \mathrm{hr})$. This is nomal. A high level signal from any RAM unit drives the blue rotating beacons on the building roof. RAM unit power is in the operational Interlock Chain (see Guide to Interlocks and Interlock Chains Section D-12). The RAM units at RUNS-II are maintained and calibrated by Plant Engineering Personnel. 
RAW Units at RenNS-II

\section{BAY Unit}

Left Machine Room

Left Target Room door

Left Target Room door Fence position

Power Supply Room rack

Hot Work Room door

Target Storage Vault door

Right Machine Room

Right Target Room door Fence position

${ }_{\mathrm{L}}=$ Local, $\mathrm{R}=$ Remote
Readont I/R*

L

L

L

R

L

R

L

R

L

R

L

L

R
Tyge of Alarm

Lights (3)

Lights (3)

Lights (3)

Light: and audible

Lights (3)

Light and audible

Lights (3)

Light and audible

Lights (3)

Light and audible

Lights (2)

Lights (2)

Light and audible 


\section{Section F}

Guide to Neutron Source Alarms

February 22, 1.985 
I. INTRODUCIION

This guide describes the alarms in use at klts-II to indicate abnormal conditions (i.e, alarms are not activated under normal conditions). All alazms have at least indicator lights, same may also have audible gignals also. To the extent possible (excertions are noted), the following convention holds

amber light - abnormal canditions but no interlock function red light - abnormal condition with interlock function 〈i.e. tive alaming element is also an interlocking element; see Guide to Interlocks and Interlocis Chains, Section D)

Because each alarm indicates an abnormal condition, the cause of each alarm should be investigated and renedial action taken. Alartis should not be left in the activated mode for long periods unless there is good reascon and such reason is noted in the $\log$ book. 


\section{Terminal Alarms}

ALARM AIARY EUNCDION

TYPE OF AIARM

TC 1

Fore purne fore vac bad

Light (red)

TC 2

Source turbo fore vac bad

Light (red)

MC 3

Ion source pressure bad

Light (red)

TC 4

Plenum turbo fore vac bad

Light (red) 
Neutron Source Alazms (Control Room)

ALAFU

TC 6

TC 7

TC 8

IC 9

TC 10

TC 11

TC 26

TC 27

3 pgi over pressure

Decel Alarm

Isolation transformer Haefely isolation transformer
over temperature Terpetature high

High voltage Haefely high voltage
off

Target water

flow high

Hunidity high

Temperature high

Stack flow velometer* Main stack air flow

Flow alarm*

Left. Target Room

Right Target Room

Hood \& storage vault

Pl Alarm*

Tritium Monitors*

$1-9,11$

low

high
Low

ATARY EXNCTLON

Mezz forepump pressure bad

TP1, TP2 fore vac bad

V18 to V23 pressure bad

TP3, TP4 fore vac bad

Target pressure bad

Target roughing line pressure bad

Target outer groove pressure bad

Target inner groove pressure bad

Mezz forepump exhaust pressure high

Ion source Decel current high

Target $\mathrm{H}_{2} \mathrm{O}$ flow

$>20$ gpm

Machine Room

Humidity high

Machine Room

Termerature high

Air flow low

Pump for stack tritium

Monitor not on

Exceeded low alarm setting

Exceeded high alarm setting
TWPE OE ATAAR

Light (anber)

Light (red!

Light (red)

Light (red)

Light (amber)

Light (red)

Light (amber)

Light (amber)

Light (red)

Flashing light (amber)

\& audible

Flashing light (amber)

\& audible

Flashing light (amber) \& audible

Flashing light (amber)

\& audible

Flashing light (amber)

Flashing light (amber)

Light (amber)

Light (amber)

Flashing light (amber) \& audible

Light (amber)

Flashing light (amber)

$\&$ audible

Ilight (amber)

Light (anber)

Flashing light (amber)

\& audible 
Pressure transducer*

Low

Bigh
Tritiun scrubber input pressure low Tritium scrubber input pressure high

as previous one

Mass flow outside linits

Scrubber flow

Low or high

Scrubber blower

suction

Low or high

Scrubber HIR tentp Low or high

Sample short Left or right

Gamma system power

Low coolant flow**

Compressor

malfunction**

Low coolant level**

Blower suction

Outside limits
TOW compressor failure

Tow chiller water

Level low
Light (amber

Light (red)

Flashing light (red)

\& audible

Lights (amber)

\& audible

Liyghts (amber)

\& audible

Lights (amber)

c audible

Lights (amber)

\& audible

Lights (amber)

\& audible

Light (red)

\& audible

Light (amber)

Iuight (amber)

Light (amber)

* Alarms on tritium monitor map are not interlocked

**Alarms on target water chiller control 
Section G

Periodic Check and Maintenance schedule

February 22, 1985

: 
I. This procedure defines general guidelines for frequency of periodic status checks and/or maintenance of the RINS-II neutron sources. These are guidelines only and do not preclude more frequent or "as needed" maintenance and checks. Unless otherwise noted, these are the responsibilities of the crations staff. AlI checks and/or maintenance should be recorded in log book and initialed. An initialed log book entry is required each day, each shift.

II. Daily

A. Status of all vacuum systems

B. Status of all doors and gates

C. Status of tritium gcrubber and tritium monitor alarms

D. Haefely motor generator hours for lubrication needs

E. Status of exhaust fan flows (particularly main stack)

F. Status of any interlock "buggers" in system

III. Heakly

A. Change ion source filament as needed.

B. Surge resistor and diode strings on Haefely (Electroncs Techricians)

c. Target oil mister leveis and pressure

D. Target chilled water level

E. Target differential pump oil level

F. Target differential purp belts

G. Tritiun scrubber sieves

H. Ion source gas bottle pressure

I. Accelerator colum water hoses

J. Mezzanine and terminal turbo and forepump/biower oil levels

K. Tritium monitor alarm set points (H/S Technician)

L. Status of leak checkers 
IV. Monthly
A. Baefely isolation tranaformer fans
B. Targec chilled water make-up cartridges
C. High voltage pit sump pumps
D. LCW water filters
E. Target chilled water filters
F. Target chilled water skimmer cartridges
G. Column resistors (Electronic Technicians)
H. Change differential puIP hoses during target change.

V. Semi-Annually

A. Turbo pump oil change

VI. Annually

A. Turbo pump bearing change (Mechanical Technicians)

VII. At Tarqat 'onange
A. Target room turbo oil level
B. Condition of differential punp hoses
C. Target air and water lines
D. Beam current pick-off lead
E. Position of PRC counters and ion chanbers
F. Carbon fiber composite target ring
G. Oil mist collector level 
Section H

Loss of Vacuum

February 22, 1985 
Recovering From Loss of Vaculua at fenis-II

This procedure describes general guidelines to aid in the restart of vacuum systems after a neutron source vacuum $106 s$.

I. In the case of a vacuum burst in which the beam line gate valves close (V5, V18, v23), and fore vac valves (v16, $\left.v_{17}, \mathrm{v19}\right)$ close the following steps should be taken:

A. Assume the possible leak is due to water.

B. Scram the Arc (if not already done by autamatic shutdown of terminal by vacuum burst).

C. Immediately stop target rotation.

D. Secure target chilled water system after target rotation stops.

E. Turn off LOW to mezzanine (V12, V14 on water map).

F. Turn off target cart water (V77, vis on water map).

G. Assuming terminal has also gone to air, proceed to gecure terminal by first shutting off ion source water (VI and V2 terminal water map).

II. The two most probable sources of water leaks are in the

A. Target area

B. Ion source area in the high voltage terminal

III. To determine the possible causes of a vacuum problem, examine all exterior components such as ion gauges, windois, etc. in the terminal and on the mezzanine for evidence of: cracking. This may also show evidence of water inside the vacuun system. Examine the terminal and mezzanine iorepump/olowers for evidence of "frothy" oil which may indicate water in the oil. If this examination reveals no evidence of problems, the recovery of the racuuth system should be undertaken in gtages. In all the following discussions, recall certain valves are interlocked to TC gauges which will require resetting limits to open the valves. 
IV. ACCEIBRATER TUBE VACUIM RESTART

A. Under the assumption that the vacum burst also closed all fore valves determine that the fore vacuums for the mezzanine turbo pumpe are acceptable (TC6 and TC7). If they are not, this may indicate an external vacutum leak between the mezzanine forepump/blower and the fore vacuum valves or water in the fore lines. Large amounts of water in forelines will be evidenced by chilling or even frosting of forelines and/or bellows.

B. If the fore vacuums are acceptable proceed to roughing bypuss on the accelerator tube (open v15).

c. If TC7 indicates the accelerator tube is pumping down, you can proceed to full roughing (TC7 reset V15 closed, V16 and V17 open.

D. TC7 should orop to about 200 milli-torr.

E. At this point, the mezzanine turbo pumps (IPl and TP2) may be started.

F. Reset limit on TC7.

V. TERHINAL VACUTM RESTART

A. Make sure terminal fore vacuums are acceptable (TCl and TC4).

B. Kake sure V4 is closed.

c. Open roughing bypass valve (v1). Note this opens both v1 and VIA.

D. If TC2 indicates bend magnet chamber is pumping jown you can proceed to full roughing (close $v 1$ open v2 and v12).

E. After TC2 reads $<200$ milli-torr, the terminal turbo pumps can be restarted.

F. Reset linits on $\mathrm{TCl}, \mathrm{TC2}$, $\mathrm{TCA}$.

G. Remember the ion source (above V4) in terminal is not yet punped out. The ion source can be pumped out in the usual manner. 
VI. REAM TRAKSFORT SYSTEM VACULM RESTARI BEMWEEN V18 AND V23

A. Close V18 and $v_{23}$ if not already cloged.

B. Close V16 and V17 (fore vac valves for TP1 and TP2) if not already closed.

C. Close V32 and V33 to the target if not already closed.

D. Close V19 (TP3 and TP4 fore vac valve) if not already clcsed.

E. Vacuum gystem between $\mathrm{V} 18$ and $\mathrm{V} 23$ is now isolated.

F. Open V33 (beam transport system roughing bypass valve).

G. After TC8 comes down to about 5000 milli-torr close v33 and open vis (fore vac valve).

H. After TC8 and TC9 drop below about $200 \mathrm{milli-torr,} \mathrm{TPl} \mathrm{and}$ TP2 can be turnea on.

I. After a few minutes, IG4 and IG5 can be used to determine condition of vacuum.

J. Reset limits on TC7 and TCB.

VII. RESTART TARGEP VACUTM SYSTEM

A. V18 and V23 are assumed to be cloged.

B. Close V16, V17, and V19 (fore vac valres to TP1 - TP4). Ensure that v30 (target vent valve) is closed.

C. Open V32 (target roughir, by-ress valve).

D. If TC10 and TCll drop to about: 5000 milli-torr, close V32 and open $\mathrm{V} 22$ (target roughing va?ve).

E. 1 Clo (target TC gauge) should drop to $<200$ milli-torr. Note this may take several minutes.

F. After TC10 < 200 mil.li-torr, close V22 and open V19. Inen open V23 while holding V23 bypass button. If V23 does not stay open, check that IG4 and IG5 filaments are on. If not, tum filaments on and try v23 again.

G. When V23 stays open, reopen V16 and VI7 for TP1 and TP2.

H. Remember that during the period v16, V17 and v19 are closed, there is no backing for TP1 - TP4. Be aware of the pressures at TC7 and TC9 if TPI - TP4 are running.

I. Reset limite on all TC gauges as necessary. 
Section I

Use of Anti-Contamination Clothirig

February 22, 1985 
Use of Anti-contamination Clothing at RISS-II

This procedure defines requifements for anti-contamination clothing when working in the FWA at RINS-II. Persons working under this procedure must be faniliar with the Rmis-II FSP or have the permission of the Health and Safety Technician.

I. TAREET ROCM ENREY (authorization, two persons and $l 0 \mathrm{~g}$-in reguired)

\section{Before entering Change Shed}

A. Foot Cover

1. yellow booties inside; attached bunny ouit booties next; hard-soled plastic booties outside (preferred method).

2. yellow booties inside; attached bunny suit booties next; yellow booties outside.

B. Body and head cover

1. Bunny suit with hood.

c. Hand cover

1. rubber gloves with wrists taped; a second pair of rubber gloves.

D. Face cover

1. respirator* when target system is opes or as deened necessary.

At the change shed

E. Add a second pair of rubber gloves if not already done.

F. Add an outside pair of booties if not already done.

G. Change outer untaped rubber gloves frequently if hands are in contact with high tritiun-contaminated parts (every 10-15 minut,es).

H. After conpletion of work, enter Change Shed and remove outer rubber gloves. Use inner pair of gloves to remove outer booties and bunny suit. Remove inner yellow booties, then gloves one at a time stepping out with the bare shoe onto rlean asphalt area. unprotected shoes should not contact floor of ti: Change shed. Dispose of all protective clothing properly.

I. For Target Rocm entry for minor adjustments etc., with target or vacuum system not open one person may enter the Target Room dressed as noted above provided a second person observes from the entrance to the Target Room. The observer must wear lab coat, rubber gloves and booties. 
J. To enter the Change Shed or concrete pad outside the Target Rocm requires booties, gloves and a lab coat. One person is sufficient. The Target Rocm door can be closed but must not be copened.

R. Check hands and feet with hand and foot counter. Shoes must be swiped before leaving the RMA. If shoes camot inmediately be checked, white booties must be worn until such time as they can be checked. Note the hand and foot counter does not check for tritium, but is sensitive to TiT/ $_{2}$ particulato.

II. BOT WORK POMN ENIRY (authorization, two persons and $\log$-in required)

A. For work in the hood on parts from the target or vaculim systems, the anti-contamination clothing reguirements are the same as for Target Roon entry.

B. For Hot Work Room entry, which dces not invo'" o work in the hood or with targets, the required anti-contanination clothing is lab coat, booties, and rubber gloves. In this case, one person may observe from the entry door and is not required to wear protective clothing.

c. Remove aJ I protastive clothing and dispose of properly in appropriate area of Hot Work Roam before entering a defined clean area.

D. Hand and foot checks are required before leaving the RMA (see section I.R above).

III. BOX CAR ENIRY (authorization, two persons and log-in required)

A. The required anti-contamination clothing is lab coat, booties and rubber gloves.

B. As for Hot work Rocm entry, one person may observe from the door without protective clothing.

C. Remov 2 and dispose of all protective clothing at exit of boxcar.

D. Hand and foot checks are required before leaving the RMA (see section I.K above).

IV. WORK ON VACUTM SYSTEMS IN MACHINE ROOM

A. For the initial opening of any vacuum system (except the ion source), the reguired anti-contamination clothing is bunny suit, rubber gloves, and respirator.* For extended periods of work, the rubber gloves should be changed frequently.

B. Booties are required as needed.

C. For ion source work, a lab coat and gloves are required.

D. Dispose of all protective clothing properly.

E. Check hands and feet with hand and foot counter. 
V. OTHER WORR

A. Anti-contamination clothing requirements for other work will be determined on the basis of need after consultation with the Health and Safety Technician. Exwever, use of gloves and lab coats is encouraged for all work on an BdN'S-II neutron source as a precautionary masure.

B. Any protective clothing must be disposed of properly.

C. Hand and foot checks may be required.

VI. TECHRIQUE

A. When leaving a protective clothing area, observe the "change line" carefully. Don't step on the dirty side with your shoes on or the clean side with dirty booties.

B. When removing protective gloves, coats and bunny suits, turn them inside out so as to contain any possible contamination more effectively.

* Respirators which can be used are:

A. 3id 9940 (preferred)

B. Norton I0030S, 10030M

All respirators must be fitted to determine proper size and sealing. All users must have a current laboratory training and fitting card. For hazardous non-routine operations a Health Physics and/or Industrial Hygienist will be consulted on breathing air protection. iNon-disposable respirators can be worn after consultation with the Health Physicist concerning type. 


\section{Section $\mathrm{J}$}

Tritium Scrubber Maintenance/Checks

February 22, 1985 
I. INIFODUCIION

This section describes the routine maintenance/checks to be performed on the tritium scrubber system to engure proper operation. There are two scrubber unitg; one on-line, one on standby. A simplified scherratic diagram is also given. Complete schenatic diagrams are posted on tritium scrubber unit 11 in the Tritium Scrubber Room ( $\mathrm{Pm}$ 1202) and at the tritium monitor rack in the Power Supply Rocm (Fm 1204).

The purpose of the tritium scrubber is to renove tritium fram the vacuum system exhaust before the exhaust is sent to the main stack for release into the atmosphere. The tritiated hydro-carbon vapors in the vacuum gyatem exhaust and bleed air (2 SCFH) from each terminal are first passed through particulate filters. They then pass through a catalytic converter which converts the tritiated bydrocarbons to tritiated water and/or water vapor. (The bleed air is used to improve the catalytic conversion process). After passing through a cooling loop, the tritiated water passes through a series of two molecular sieve beds which trap the moisture. Further air is mixeo with the scrubbed exhaust before it is sent to the main stack for release.

A tritium monitor samples the processed exhaust between the two molecular sieve beds. This is used along with moisture indicators in the molecular sieves to indicate beginning of breakthrough of the first molecular sieve.

During pump-down of a vacuum system, various monitored functions of the tritiun scrubber will alarm. The alarms (for the unit on line) which will activate under purp-down conditions are:

1. Scrubber Input Pressure - high alarm

2. Scrubber Flow - high alarm

3. Scrubber Blower suction - low alarm

After a short period (about 2-3 minutes) these alarms will clear. These alarms are not activated under normal operating conditions. Therefore, an alarm under these conditions indicates a possible scrubber malfunction and should be investigated at once. 
II. MOKIINAL TRITIUH SCRUBBER MONATORED READINCS

The nominal readings for the monitored fumctions on a tritium scrubber (when in operation are:

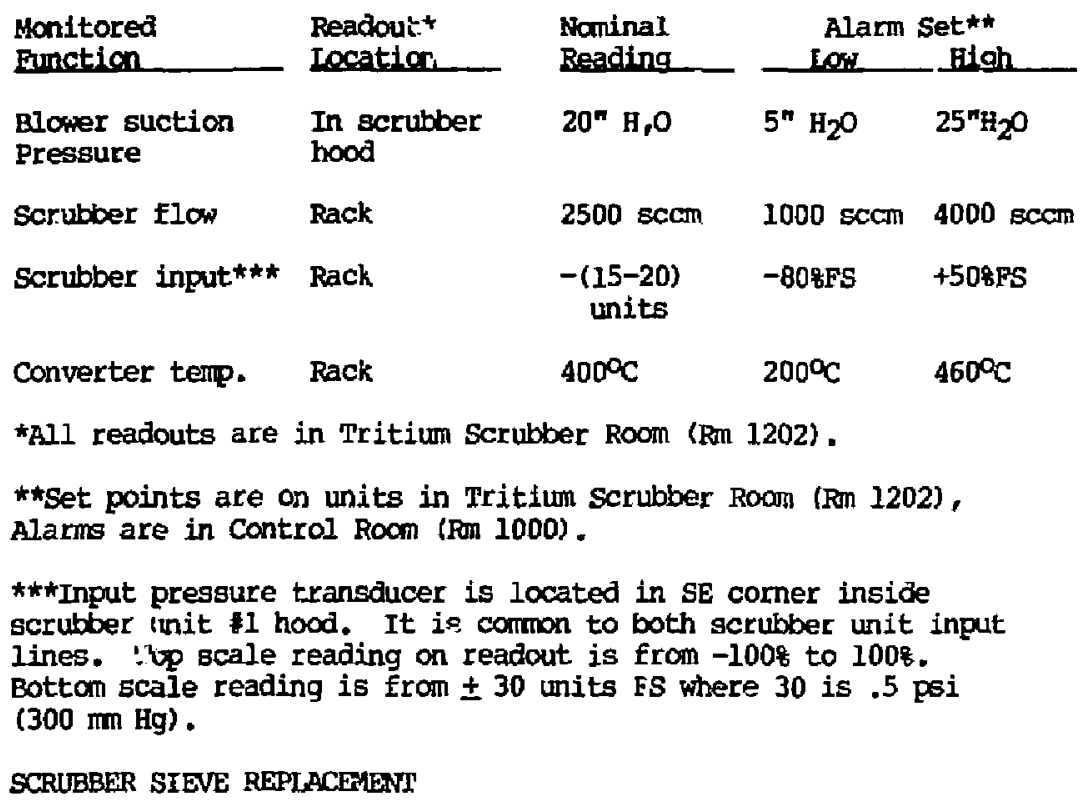

When a tritium monitor sampling a scrubber unit goes into alarm or when the moisture indicators in a sieve turn pink, this indicates the need for changing of the sieve. The following procedure should be used:

1. Lab coats and gloves must be used when working in a scrubber hood.

2. Valve in new sieve if appropriate (i.e. sieve to be changed is one of the two parallel sieves). Valve off eieve to be changed. (If sieve to be -ranged is the final sieve in the on-line scrubber, you should firet put standby scrubber on line. Then valve off scrubber and sieve to be worked on.)

3. Remove and immediately cap used sieve.

4. Ingtall new sleve.

5. Put used sleve in IVC pipe container and glue shut.

6. Ensure that you have normal readings on the on-line scrubber.

7. Place Puc-contained Bieve in the labelled 55-gal. drum in FNA and recover c'rum.

8. If necessary, recheck for proper readings at scrubber and that appropriate valves are in proper position. 
TRITIUM SCRUBBER DIAGRAM * INPUT [PUMP EXITAUST \& BLEED AIR]

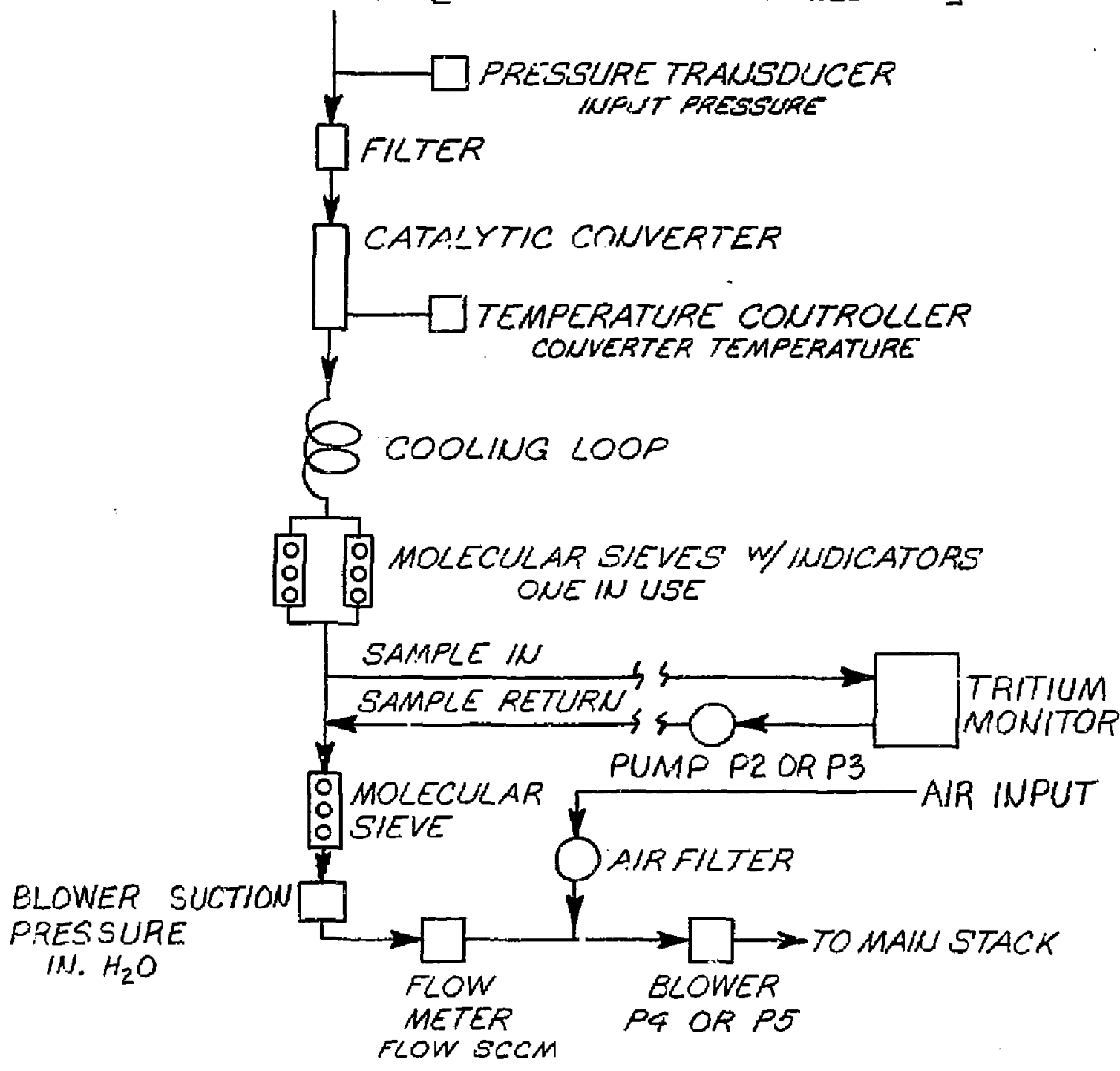

* NO VALVES ARE SHOWN 
Section $\mathrm{K}$

TYpical iveutron Source Start-up Procedure

February 22, 1985 
$\mathrm{k}-1$

\section{INIRODUCTION}

In this section a typical start-up procedure is given for the Ieft Neutron source. A simillar procedure is used for the Right kiutron source except where reference is made to specific rack or panel locations.

This procedure is not intended to be of the "check-list" type. It jz rather intended to list "normal or usual" steps taken in starting a Meutron source. 
Control Reom

\begin{tabular}{|c|c|c|}
\hline$C-04$ & $\begin{array}{l}\text { Read } \log \text { book } \\
\text { Check IG1 and IG2 } \\
\text { Check source and plenum turbo pumps "ON" }\end{array}$ & \\
\hline $\mathrm{C}-0 \mathrm{I}$ & $\begin{array}{l}\text { Check vacuum system gauges } \\
\text { Check turbo punps "ON" } \\
\text { Remove key a fron key lock }\end{array}$ & $\begin{array}{l}\text { IG3, IG4 and IG5 } \\
\text { TPl, } 2,3 \text { and } 4\end{array}$ \\
\hline
\end{tabular}

rachine Bogm

Terminal

Open door latch and use key to open door Bring key with you when entering

Make sure ground hooks are in place

Turn $c$ breakers 2 and 4 in panel 44049-9

In rack 55 turn on all labeled breakers

Turn on water $\mathrm{V} 1$ and $\mathrm{V} 2$ (HV term. water control map)

Check that all flow meter lights made up

Open gas valve v8. Re-check IG1 and IG2

Open beam line valve $\sqrt{5}$

Turn on ali breake:s for cober P.S.

Push system "ON" button on Cober and wait for current limit

light to go out

Push "RESET" button, "READY" comes on:

Insert key in "Haint. By-pass Mode" lock, turn key and hold down

"Transfer" button and key "Operate ready" lock

Remove ground hook and hang on interlock hanger by door

close terminal door and lock

Hove ladder to a spot by entry door

Mezzanine

Rey "run/safe" box

Cloge and latch gate upon leaving mezzanine

Move mezzanine latter to west wail near entry door

H.V. Pit

If the red light $o$ the $S E$ corner is not flashing:

Go down the ladder on south wall and check for perscrunel. If clear, key "run/safe" on north wall then key "run/safe"

on east wall. Leave pit and close trap door

Closing Kachine Rocen

Rey SE "run/safe" box

Move ground hook from base of teminal (w corner) and place on microswitch hook in the floor

Rey Sw "run/safe" box

Hove ground hook from ow tower \& place on microswitch hook by enticy door

Rey "run/safe" tox by entry door

CFIDCK THAT NO ONE IS IEFT IN MACHINE ROON-SEOUT: "CLEAR THE PIT"

Close entry coor and secure latch 


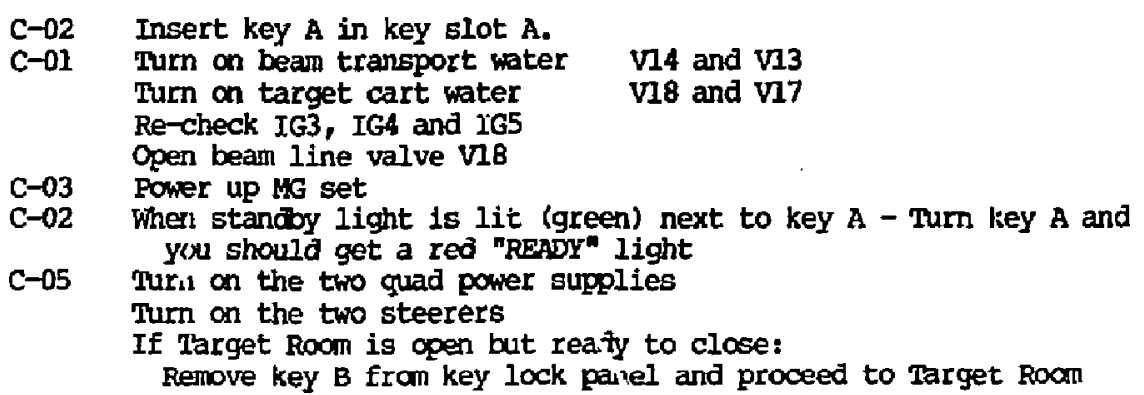

TARGET ROOM

Note: Booties, bunny suit, \& rubber gloves are required for entry into Target

Room (see Section I for authorization and clothing requirements) Check to see that no one is inside With Target Roon clear, insert key and close door Close latch and remove key Insert key in gray box and make up interlocks Key "safe/run" box at the door Return to Control Roon with key

DONIROL ROOM

C-02 Insert key B in key slot B

Target Water System

C-02 Push TOW return valve button, TOW supply valve button and oil

mist button "ON"

C-09 If not already "ON", turn on pump and either compressor 1 or 2

C-02 Check flow: ghould be $\sim 12-1.5$ gam with no rotation Target Rotation

Enable target controller

Verify rotation and scan limits

With rotation control in manual increase rotation speer to $22 / 3$ of final valve and then switch to the auto mode for speed control. Observe that appropriate speed has been reached and is being controlled.

Scan can be started as needed 
Ion source start-up

C-04 Push source gas button on remote control panel. Button should light up

Also, power, arc scram and nlinging target buttons should be lit

C-04 Check IV an left side of screen that chain and gas lights are lit. Also on the botton of the screen that the wurbos and v5 lights are lit

C-04 The following components should already be set: source lens (n.75 volts), bending magnet ( 6.3 amgs and steerer 11 . $( \pm 3)$.

C-04 Rermote Conticol panel

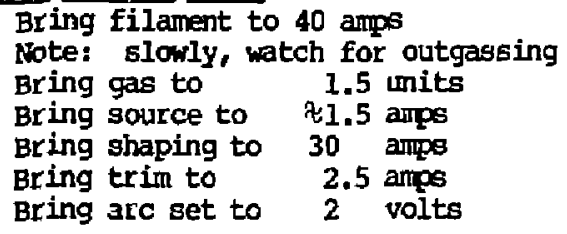

Striking Arc

c-04 In-scram arc by pressing the arc scram button and the arc should strike

If the arc doesn't strike, then press arc soram and raise the filament or gas up a little and try again

c-04 Scram the arc and push decel button on (light 1it). Paise decel to $\approx 3 \mathrm{kV}$ and ext. volts to $232 \mathrm{kV}$.

Bring H.V. on

C-02 Tum key B to "ON" (red ready light)

C-03 Rey on HV control panel

Turn on KG set (start lights)

Press manual restart button

After a "ON" light for MG set: press "Interlock" button

Press HV "ON" button

Turn on HV alarm 


\section{CONTROL ECOY}

Twing Beam to Target

C-04 In-scram are by pressing arc scram button tif the arc doesn't strike check "striking arc" section) Check current an plunging target 20-50 ma.

C-03 Check terminal plunging target monitor for bean centering Check with experimenter before beaming to the target Make sure the CAMAC and the ND aystem are ready to go

C-04 Remove plunging target. Check vacuum gauges. Check plenum collimator current Check target current and target collimator Use the following to maximize beam current

1. Extraction voltage

2. Source magnet

3. Shaping magnet

c. Trim magnet

5. I.S. Steerer $\$ 1$ and $\$ 2$

C-05 6. Quad mag. 1 and 2

7. Steerers 1 and 2

C-05 Check beam spot on TV for centering and size

C-05 Increase beam current slowly with arc set I until you have desired current on target

Hote: Watch for target outgassing while increasing beam current on target 
Section L

Tritium Monitoring System

February 22, 1985 
Tritium concentrations are measured at various locations throughout the Rurs-II facility (Bldg. 292). These are

$\begin{array}{ll}\text { Monitor } 1 & \text { Target storage vault/hot cell vent } \\ \text { Monitor } 2 & \text { Tritium scrubber } 12 \\ \text { Monitor } 3 & \text { Tritium scrubber } 1 \\ \text { Monitor } 4 & \text { Tritium Scrubber Room (Rm. 1202) } \\ \text { Monitor } 7 & \text { Left Target Room (Rm. 1404) } \\ \text { Monitor 6 } & \text { Left Machine Rocm (Rm. 1402) } \\ \text { Monitor } 8 & \text { Main stack exhaust } \\ \text { Monitor 9 } & \text { Right Target Rocm (Rm. 1407) } \\ \text { Monitor } 11 & \text { Right Machine Room (Rm. 1406) }\end{array}$

Monitors 2, 3 and 8 are multi-range with auto-ranging capabilit;, the ranges are $0-1 \mathrm{mCi} / \mathrm{m}^{3}, 9-10 \mathrm{mCi} / \mathrm{m}^{3}, 0-100 \mathrm{mCi} / \mathrm{m}^{3}$, and $0-20 \mathrm{Ci} / \mathrm{m}^{3}$. The others are single range units with a maximum reacing of $20,000 \mu \mathrm{Cl} / \mathrm{m}^{3}$.

\section{SCHEMATTC DIAGRAMS}

Schematic diagrans are given for the tritium monitoring units on the next pages. It should be noted that there are three different gcheratics depending on the tritium monitor unit. In practice, only unit 8 of units $1,7,8$ and 9 has the $D F O x O$ purifier section in operation. Typical flow meter readings are indicated on the schematic diagrans. More conplete diagrams of the scrubber/tritium monitor system are posted at the tritium monitor location (Power Supply Room - Rm. 1204) and on tritium scrubber unit 11 in the scrubber Room (Rm. 1202).

Each unit has high and low level alarms isee Section F, Guide to Neutron Source Alarms).

The normal settings for these alarms are (potentiometer setting).

Monitor Number

$\frac{1}{2}$
3
4
$7 *$
6
8
$9 *$
11

$\begin{array}{ll}10 \mu \mathrm{Ci} / \mathrm{m}^{3} \\ 10 & \mu \mathrm{Ci} / \mathrm{m}^{3} \\ 10 & m \\ 10 & " \\ 10 & " \\ 10 & " \\ 10 & n\end{array}$

HI.

$$
50 \mu \mathrm{Ci} / \mathrm{m}^{3}
$$$$
50 \mu \mathrm{ci} / \mathrm{m}^{3}
$$$$
500 \pi
$$$$
50 \pi
$$$$
50 \text { (n) }
$$$$
500 "
$$

50 .

* Monitors 7 and 9 have the HE set higher to avoid continual alarming from $13_{\mathrm{N}}$ activation in Target Room air during neutron source operation.

Tritium monitors 2 and 3 monitor scrubber performance and give an indication of beginning of breakthrough on the first molecular gieve (see Section J, Tritium Scrubber Naintenance). 
$L-2$

SIMPLIFIED DIAGRAM

FOR TRITIUM MONITORS $1,7,8 \xi 9 *$

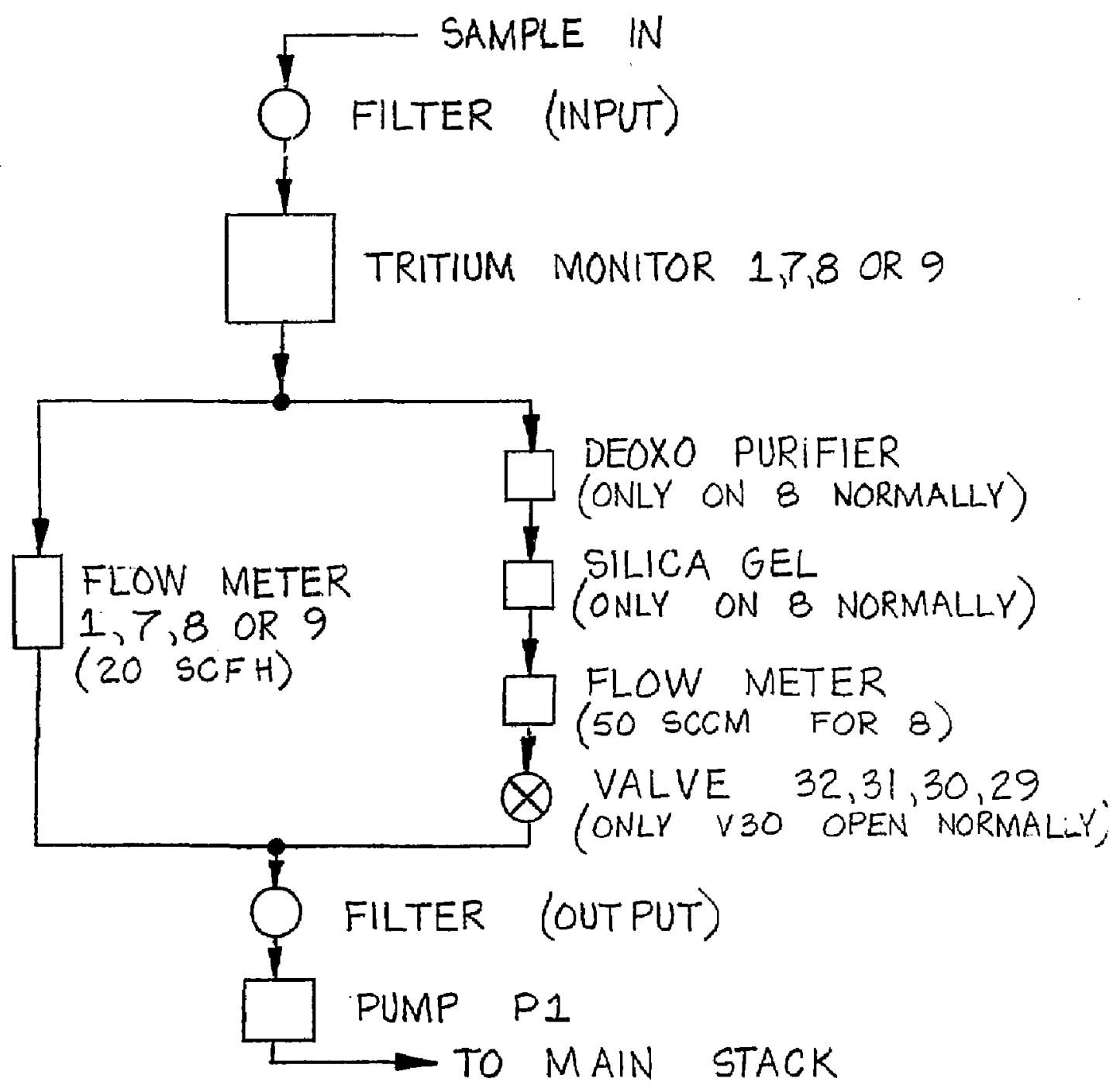

* all components except inPUT filter are LOCATED AT SOUTH END OF POWER SUPPLY ROOM (RM 1204). 
$L-3$

SIMPLIFIED DIAGRAM.

FOR TRITIUM MONITORS $2 \xi 3 *$

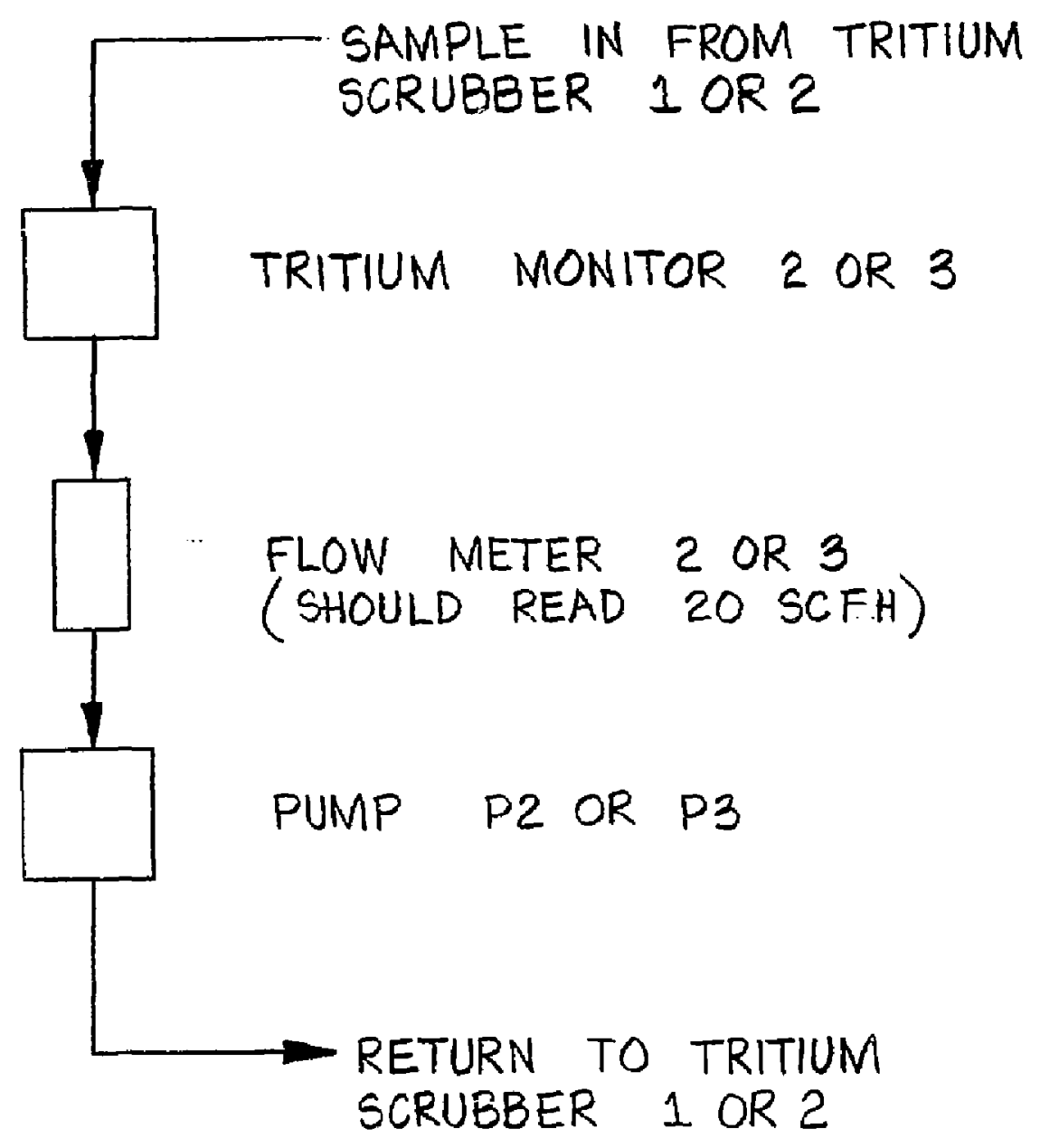

$$
\begin{aligned}
& * \quad \text { *LL COMPONENTS ARE LOCATED AT } \\
& \text { SOUTH END OF POWER SUPPLY ROOM } \\
& \text { (RM 1204). }
\end{aligned}
$$


$L-4$

SIMPLIFIED DIAGRAM

FOR TRITIUM MONITORS 4,6द11*

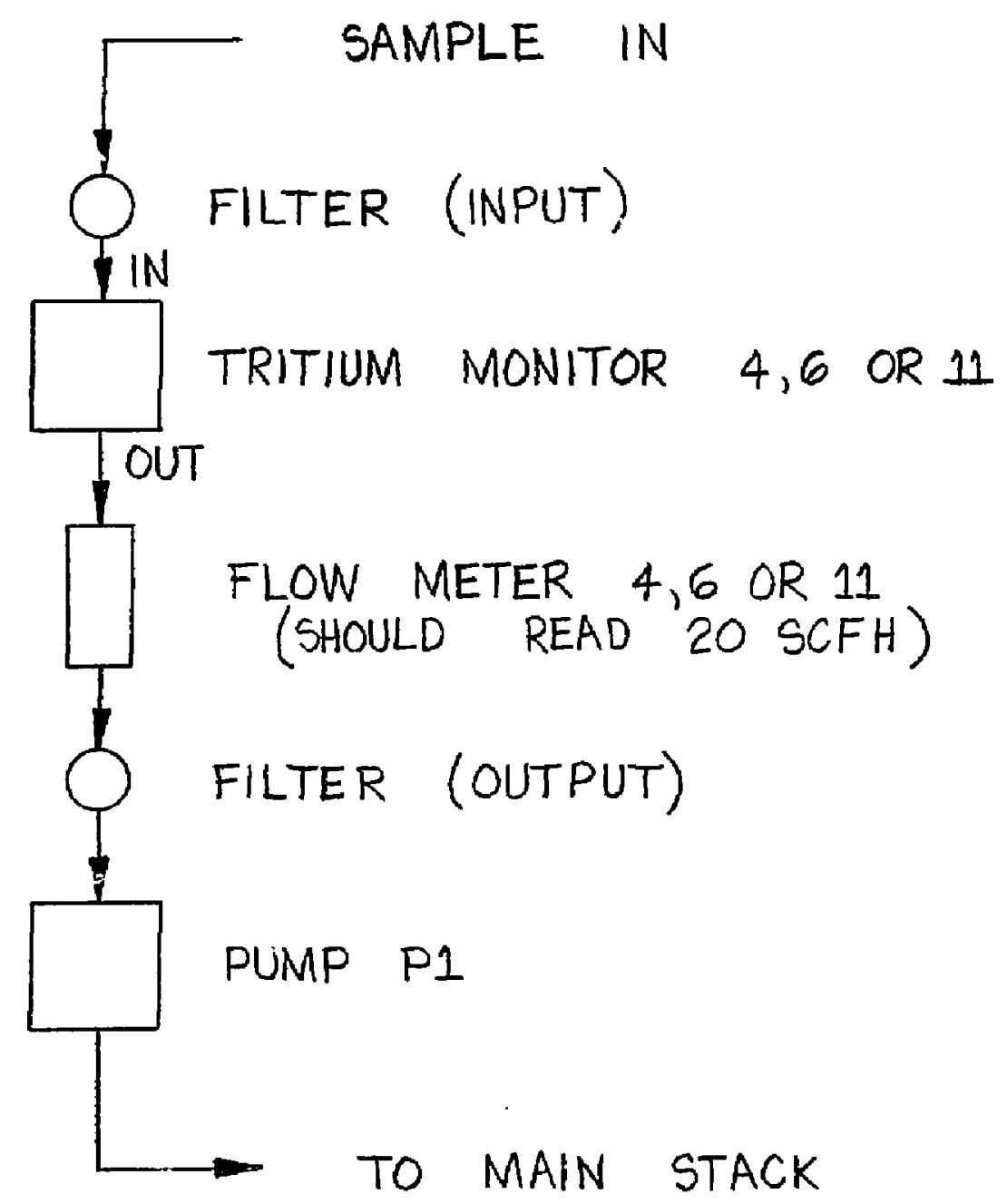

* ALL COMPONENTS EXCEPT INPUT FILTER ARE LOCATED AT SOUTH END OF POWER SUPPLY ROOM (RM 1204). 


\section{Section $d$}

Summary of Normal Readings/Settings February 22, 1985 


\section{IMIRODUCTION}

In this section we list the normal or nominal readings/settings for vacuum systeris, water flow, etc. Where applicable, readings for both standby and normal operation (producing neutrons) are given. Some variation in readings is to be expected over long periods of time. Differences can also be expected between the two neutron sources, and dependong on condition of the vacuum geal etc. However, the values given here ghould serve as useful guidelines.

We do not include readings/settings for such things as the ion source, accelerator or beam transport control parameters.

II. TERMINAL READINGS

A. Vacuum System

$$
\begin{aligned}
& \text { TC1 - TC4 } \\
& \text { IG1 } \\
& \text { IG2 }
\end{aligned}
$$

Standby

๙ 50 mtors

$\sim 4 \times 10^{-7}$

$\sim 4 \times 10^{-7}$

B. Water Flow

FS2-11

FS12

$\frac{\text { Standby }}{\text { AII Off }}$

ON
Nomal

2 50 mtork

$\sim 3 \times 10^{-5}$

$\sim 4 \times 10^{-6}$

c. Bleed Air

Bleed air should always be 25CFH (standard cubic feet/hour).

III. BEAY TRANSPORT/TARGET SYSTEM READINGS

A. Vacuum system

\begin{tabular}{|c|c|c|}
\hline & Standiby* & Normal * \\
\hline $\begin{array}{l}\text { TC6-TCl1 } \\
\text { TC26 (outer groove) } \\
\text { TC27 (inner groove) } \\
\text { IG3 } \\
\text { IG4 } \\
\text { IG5 }\end{array}$ & $\begin{array}{l}\sim 50 \text { mtorr } \\
\sim 2.5 \text { torr } \\
\sim 80 \text { mtor } \\
\sim 5 \times 10^{-7} \\
\sim 5 \times 10^{-6} \\
\sim 6 \times 10^{-6}\end{array}$ & $\begin{array}{l}\sim 50 \text { mitorr } \\
\sim 3.5 \text { torr } \\
\sim 80 \text { ntorr } \\
\sim 2 \times 10^{-6} \\
\sim \\
\sim 8 \times 10^{-6} \\
\sim .8-1.2 \times 10^{-5}\end{array}$ \\
\hline
\end{tabular}

there may be variations between Leit and Right neutron source 
B. Water Flow

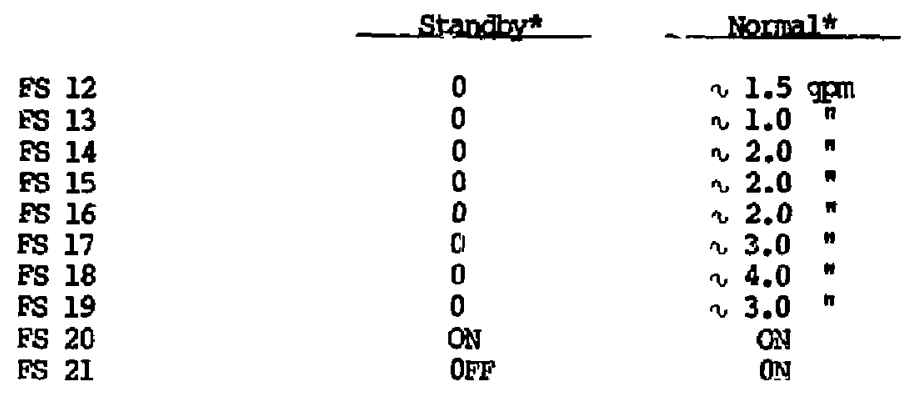

Where may be variations between Left and Right neutron source

III. TRITIUM SCRUBBER/MONITORS

A. Scrubber (normal readings)

Blower suction

Scrubber flow

$20 " \mathrm{H}_{2} \mathrm{O}$

Scrubber input pressure

Converter termerature

$\because-(15-20)$ units

$4000^{\circ}$

B. Tritiun Monitors

Flow for all units should be adjusted to 20 scFH using the flow controls. In addition, for tritium monitor 8 the flow meter in the silica gel leg should be set at $50 \mathrm{cc} / \mathrm{min}$.

C. Stack Flow Reading

The stack flow is nominally $11 \mathrm{~m} / \mathrm{s}$. This reading may vary somewhat, depending on whether the scrubber hood doors or the Hot Work Room doors are open, The stack area is $.4 \mathrm{~m}^{2}$ and therefore stack volumetric flow is $4.4 \mathrm{~m}^{3} / \mathrm{s}$.

D. Miscellaneous Readings

Isolation transformer temperature Isolation transformer current Haefely high voltage PS efficiency

unnoc

oil mister input pressure

Oil mister oil level

oil mister output presgure

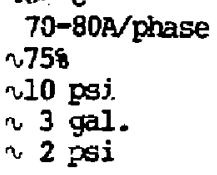


Section N

Additional Docimentation

February 22, 1985 